\title{
Knowledge and Attitudes About HPV Infection, HPV Vaccination, and Cervical Cancer Among Rural Southeast Asian Women
}

\author{
Li Ping Wong
}

Published online: 4 June 2010

(C) International Society of Behavioral Medicine 2010

\begin{abstract}
Background The incidence of cervical cancer in developing countries is high and even higher among women in rural areas in these countries.

Purpose The purpose of this study is to assess the knowledge and attitudes towards human papillomavirus (HPV), HPV vaccination, and cervical cancer among young women in rural settings in a Southeast Asia country.

Methods A convenience sample of ethnically diverse young rural women in Malaysia was surveyed. Participants were interviewed using a standard questionnaire.

Results Among the participants $(N=449)$, knowledge of HPV, HPV vaccination, cervical screening, and cervical cancer risk factors was extremely poor. The mean total knowledge score (14 items) was 2.37 ( $\mathrm{SD} \pm 1.97)$. Although many had never heard of the newly released HPV vaccine, two-thirds professed an intention to receive the HPV vaccine. Intention to receive the vaccine was significantly associated with knowledge of cervical screening and cervical cancer risk factors (OR 1.17; 95\% CI 1.03-1.33; $P=0.013$ ). Reasons for vaccine refusal were doubts about safety and efficacy of the new vaccine $(27.4 \%)$, perceived embarrassment at receiving an STI vaccine $(20.7 \%)$, and perception of not being at risk of HPV infection (20.0\%). Conclusions Providing HPV education to the rural residents is a high priority.
\end{abstract}

Keywords Knowledge $\cdot$ Attitudes $\cdot$ HPV C Cervical cancer . Young women $\cdot$ Rural residence $\cdot$ Southeast Asia

L. P. Wong $(\bowtie)$

Medical Education and Research Development Unit,

Faculty of Medicine, University of Malaya,

50603 Kuala Lumpur, Malaysia

e-mail: wonglp@ummc.edu.my

\section{Introduction}

Cervical cancer is a major cause of death in women around the world. Across the globe, over $80 \%$ of the deaths from cervical cancer occur in low-income countries and the developing world, where cervical cancer is the most common cancer in women [1]. Being the second most common female cancer in Malaysia and constituting about $12.9 \%$ of all female cancers, cervical cancer remains a significant public health problem threatening women. The Malaysian National Registry reported an average of 2000 3000 hospital admissions of cervical cancer per year, with the majority presenting at late stage of the disease [2]. It is now established that certain high-risk oncogenic types human papillomavirus (HPV) are linked to the pathogenesis of cervical cancer and its precursors [3-5]. Vaccines are now available to prevent the types of HPV that most commonly cause cervical cancer.

There is no dispute that the HPV immunization would most greatly benefit developing countries like Malaysia, and other poor countries, where women are deprived of access to effective screening and treatment programs [6]. Challenges associated with the introduction of the new vaccine in these countries include the administration cost, dissemination and access to vaccines, and social cultural aspects of immunization of a sexually transmitted infection (STI) vaccine $[6,7]$. Numerous studies have demonstrated that public awareness of HPV, perceived risks of HPV infection, and knowledge of potential health consequences of HPV infection are low [8-10]. Factors associated with the acceptability of the HPV vaccine are knowledge about the disease, beliefs about vaccines, physician recommendation, previous experience with HPV infections, perceived risk for HPV infection, perceived vaccine safety and efficacy, and perceived vaccination would promote adolescent sexual behavior [11-13]. 
It has been reported that cervical cancer is much more prevalent among rural than urban women in developing countries $[14,15]$. Similar findings were also reported in developed regions. In the USA, cervical cancer is considered one of the most deadly cancers among the medically underserved, low-income communities, and among minority women living in rural areas [16]. High rates of cervical cancer are related to a high proportion of women in these areas living in poverty, unemployment, low levels of education, and barriers to preventive screening [16-18]. This indicated that HPV immunization may be of particular benefit to the rural poor and medically underserved populations worldwide where women are deprived of access to regular cervical screening [19]. Several studies on acceptability of HPV vaccine among the underserved rural women have been reported in the literature [11, 2022]. Lack of reported data from underdeveloped and developing regions warrants further investigation, especially in the context of a high population incidence of cervical cancer in these regions and even higher incidence among the marginalized women in rural areas.

In 2006, the prophylactic vaccine against HPV was released in Malaysia and has been given regulatory approval. Although studies showed that Malaysian parents $[23,24]$ and young women [25] were generally supportive of vaccinating their children or themselves against HPV infections, there are many remaining questions. Most of these studies have been conducted in urban settings, and the published literature offers little insight into HPV vaccination intention, knowledge and attitudes of the vaccine among rural populations in Malaysia. Women in these areas are faced with limited resources, education, and reproductive decision-making. They are at the highest risk of cervical cancer yet often lack access to preventive care. These vulnerable women would benefit most from the HPV vaccination, but often will be the least likely to receive the vaccine.

The purpose of this study was to assess levels of knowledge and attitudes held by rural Malaysian women in Malaysia concerning cervical cancer-related issues, HPV infection, and the newly available HPV vaccine. Malaysia is a multiethnic and multireligious society encompasses a majority Muslim Malay population and other large ethnicities (mainly Chinese and Indian, and a large number of other indigenous ethnic groups). Introduction of an STI vaccine remains a great challenge in the predominant Muslim population where Islam is the national religion.

\section{Materials and Methods}

The survey was conducted between March and June 2008 . A two-stage sampling method was implemented. Firstly, rural villages in the states of Perak and Pahang in Peninsular Malaysia were selected, using a convenience sampling technique. In each of the selected villages, all households were visited. Households were selected to participate if an eligible participant was present at the time of visit. Women were eligible to participate if aged between 18 and 25 years, and living in the household. If a household had more than one eligible participant, one eligible member was randomly selected to participate.

A standardized questionnaire was used in the interview. The items in the questionnaire assessed knowledge, attitudes, and demographic characteristics related to HPV and cervical cancer. In the knowledge section, participants were asked a series of questions measuring their knowledge about HPV, genital warts, HPV infection, and its association with cervical cancer (six-item scale). Participants were also asked whether they had heard of cervical screening, the purpose of cervical screening, and risk factors for cervical cancer (eight-item scale). Participants were to respond with only one choice from three choices given, "true", "false", and "not sure". For each question, a correct response was given a score of one, and an incorrect or 'not sure' was scored as zero. Total scores were calculated by adding up scores of the two sections. Therefore, the possible range of obtainable scores was between 0 and 14. Participants were also asked if they had heard of the newly released HPV vaccine. Those who reported to have heard of the HPV vaccine were subsequently asked a series of questions regarding the potential benefits of the HPV vaccine.

The second section of the questionnaire assessed participants' attitudes toward receiving the HPV vaccine and reasons for acceptance or refusal of vaccination. Additionally, the participants were asked to specify what information they wanted to know about the new HPV vaccine. Prior to answering the attitude questions, participants were provided with information about HPV and cervical cancer, and the new HPV vaccine. All the questions were adapted and modified from previous studies and expert review [26-29]. The questionnaire was pilot-tested in rural settings before commencing the study. The interviews were conducted in Bahasa Melayu (the national language of Malaysia). All interviews were conducted by four female medical students, who had been trained to ensure that interviews were undertaken in a standardized manner.

Data was anonymized and analyzed using SPSS 17.0 for Windows (SPSS Inc., Chicago, IL, USA). The significance level was set at $P<0.05$. Data analysis consisted of descriptive statistics including calculation of frequencies and mean scores for the knowledge items as well as demographic variables. Data were presented as mean $(95 \%$ CI) for continuous variables. Multivariate logistic regression analyses using the 'enter' method were used to determine factors associated with attitudes towards HPV 
vaccination (namely willingness to be vaccinated and refusal to be vaccinated because of the perception of not being at risk, doubt about the efficacy and safety of the new vaccine, and embarrassment to receive an STI vaccine). The independent variables included in the models were age, ethnicity, highest educational achievement, relationship status, and knowledge scores. Goodness of fit of the statistical model was assessed with the Hosmer-Lemeshow test. The study was conducted after approval had been obtained from the Medical Ethics Committee, University Malaya Medical Center, Kuala Lumpur, Malaysia. All participants were informed about the objective of the study and written consent was obtained.

\section{Results}

\section{Participants}

A summary of the respondent characteristics is provided in Table 1. The household interview was carried out in 33 rural villages. Of the total 589 eligible participants approached, $84.7 \%$ participated $(n=499)$ in the survey. The mean age of respondents was 21.17 years $(\mathrm{SD} \pm 1.89)$. The majority of respondents were Malay (42.3\%) followed by Chinese $(29.2 \%)$ and Indian $(28.5 \%)$. The majority $(95.3 \%)$ had attained at least secondary education. Only $2.0 \%$ were married, more than half $(58.4 \%)$ reported they were not dating, while only $0.4 \%$ noted that they were living with a steady partner.

\section{Knowledge and Awareness}

Of the participants, only $11.6 \%(n=52)$ had heard of HPV and only $7.8 \%(n=35)$ had heard of the newly released HPV vaccine. Of those who had heard of the HPV vaccine, the majority $(45.7 \%)$ heard about the vaccine from friends, followed by public media $(31.4 \%$ television, $20 \%$ newspapers, $17.1 \%$ radio, and $10.0 \%$ magazines). Participants of 21 years of age or older $(51.4 \%)[\chi 2(n=35, \mathrm{df}-1)=1.57$, $p=0.14]$ and higher educational level $(97.1 \%)[\chi 2(n=35$, df -1$)=0.28, p=0.50$ ] were more likely to report that they had heard of the vaccine. Respondents who were not in a dating relationship (62.9\%) were more likely than those in dating relationship or married to report that they had heard about the HPV vaccine [ $\chi 2(n=35, \mathrm{df}-3)=8.25, p=0.04]$. Lack of knowledge of the HPV vaccine was found even among those that reported to have heard of the vaccine. When they were asked a series of questions regarding the potential benefits of the HPV vaccine, the majority answered "not sure" or chose the incorrect answer.

The mean score for knowledge of HPV and genital warts for all participants $(n=449)$ was only 0.45 out of $6(\mathrm{SD} \pm$
Table 1 Demographic characteristic of respondents $(n=449)$

\begin{tabular}{lc}
\hline Characteristic & $N(\%)$ \\
\hline Etnicity & \\
Malay & $190(42.3)$ \\
Chinese & $131(29.2)$ \\
Indian & $128(28.5)$ \\
Religion & \\
Islam & $191(42.5)$ \\
Buddhist & $118(26.3)$ \\
Hindu & $124(27.6)$ \\
Christian & $16(3.6)$ \\
Current/highest educational level ${ }^{\mathrm{a}}$ & \\
No formal school & $2(0.4)$ \\
Primary school & $19(4.2)$ \\
Secondary school & $358(79.7)$ \\
College/University & $70(15.6)$ \\
Relationship status & \\
Not dating & $262(58.4)$ \\
Dating, no steady partner & $96(21.4)$ \\
Steady partner, not living together & $80(17.8)$ \\
Steady partner, living together & $2(0.4)$ \\
Married & $9(2.0)$ \\
\hline
\end{tabular}

All vales are based on participants self-reporting

${ }^{\text {a }}$ Value do not sum up to $100 \%$ due to rounding

$0.81 ; 95 \%$ CI $0.38-0.53)$. The majority $(79.7 \%, n=358)$ was not sure that HPV is related to cervical cancer and only $15.8 \%(n=71)$ provided the correct response. Mode of transmission of HPV was only known by $8.5 \%(n=38)$, while $89.3 \%(n=401)$ indicated that they were not aware that HPV infection is common.

Likewise, knowledge of cervical screening and cervical cancer risk factors were also poor, with the mean score of only 1.92 out of $8(\mathrm{SD} \pm 1.68 ; 95 \%$ CI $1.76-2.07)$ for all participants $(n=449)$. Only $29.6 \%(n=133)$ had heard about the Pap smear test. Only $11.4 \%(n=51)$ correctly explained the purpose of the Pap smear test, and $78.6 \%(n=353)$ were not sure about its purpose. A small minority of the participants had the misperception that the Pap smear test is used for the treatment for cervical cancer (5.1\%) or to test for sexually transmitted diseases $(5.1 \%)$. Only slightly above half $(54.1 \%, n=243)$ were able to identify multiple sexual partners as a risk factor for cervical cancer. Knowledge of multiple sexual partners as a risk factor for cervical cancer was not associated with status of relationship and educational level. However, a slightly higher proportion of non-Malay participants $(51.9 \%)$ correctly identified this risk compared to Malay participants (48.1\%) [ $\chi 2(n=449, \mathrm{df}-2)=9.91, p=0.007]$.

Across the sample $(n=449)$, the mean total knowledge score (14 items) was only 2.37 ( $\mathrm{SD} \pm 1.97$; 95\% CI 2.19 
2.55). The overall knowledge score was positively skewed (range $0-10$, median 2 , interquartile range $0-4$ ). Table 2 shows the association between participants' demographic characteristics and mean knowledge scores. There were significant differences in overall mean knowledge scores among the three ethnic groups. Participants with postsecondary education had significantly higher overall mean knowledge scores. Participants with highest scores were those who were married and in a steady dating relationship.

Attitudes

Approximately $65 \%$ of participants indicated the intention to receive the HPV vaccine. A total of $27.4 \%$ refused vaccination because of doubts on the safety and efficacy of the new vaccine, embarrassment about receiving an STI vaccine $(20.7 \%)$, and the perception of not being at risk of HPV infection (20.0\%). Table 3 presents the associations between predictor variables and attitudes towards HPV vaccination. The model reveals that intention to receive the HPV vaccine was significantly associated with knowledge of cervical screening and cervical cancer risk factors. However, no significant association was found between intention to receive the HPV vaccination and age, ethnicity, educational level, relationship status, and knowing someone with cervical cancer. Perceived embarrassment of receiving an STI vaccine was significantly associated with age, whereby younger participants were more likely to report shame. The Hosmer and Lemeshow Goodness-of-Fit tests were not significant, which indicate a very good model fit (Table 3). The study also showed that approximately $74 \%$ $(n=330)$ indicated that if the physician would recommend vaccination, they would likely accept the vaccine.

Information Needs

When participants were asked to specify what information they wanted about the new HPV vaccine, near $90 \%$ of the study participants reported they required more information about why a person needs to be vaccinated and who would need to be vaccinated $(85.3 \%)$ and potential risks or side effects of HPV vaccination (85.7\%).

Table 2 Association between demographic characteristics and mean knowledge scores

\begin{tabular}{|c|c|c|c|c|c|}
\hline \multirow[t]{2}{*}{ Characteristic } & \multirow{2}{*}{$\begin{array}{l}\text { All } \\
\text { respondents } \\
(n=449)\end{array}$} & \multicolumn{4}{|l|}{ Mean score $(95 \% \mathrm{CI})$} \\
\hline & & $\begin{array}{l}\text { Knowledge of HPV } \\
\text { and genital warts } \\
(0-6 \text { items scale })\end{array}$ & $\begin{array}{l}\text { Pap smear and cervical } \\
\text { cancer risk factors } \\
(0-8 \text { items scale })\end{array}$ & $\begin{array}{l}\text { Total knowledge } \\
(0-14 \text { items scale })\end{array}$ & $P$ value \\
\hline \multicolumn{6}{|l|}{ Age } \\
\hline$\leq 21$ years & 263 & $0.46(0.37-0.56)$ & $1.87(1.66-2.08)$ & $2.33(2.10-2.57)$ & \multirow{2}{*}{$\begin{array}{l}P=0.65 \\
t=-0.449\end{array}$} \\
\hline$>21$ years & 186 & $0.44(0.32-0.56)$ & $1.98(1.74-2.21)$ & $2.42(2.13-2.71)$ & \\
\hline \multicolumn{6}{|l|}{ Ethnicity } \\
\hline Malay & 190 & $0.51(0.39-0.63)$ & $2.12(1.88-2.37)$ & $2.63(2.34-2.92)$ & \multirow{3}{*}{$\begin{array}{l}P=0.00^{* *} \\
F(2,446)=9.8^{\mathrm{a}}\end{array}$} \\
\hline Chinese & 131 & $0.32(0.20-0.45)$ & $1.42(1.17-1.67)$ & $1.74(1.44-2.04)$ & \\
\hline Indian & 128 & $0.51(0.36-0.66)$ & $2.12(1.82-2.42)$ & $2.63(2.28-2.97)$ & \\
\hline \multicolumn{6}{|l|}{ Educational level } \\
\hline Low Education & & & & & \multirow{3}{*}{$\begin{array}{l}P=0.013^{*} \\
F(2,47.69)=4.74^{\mathrm{b}}\end{array}$} \\
\hline Primary school & 21 & $0.48(-0.01-0.97)$ & $1.81(1.16-2.46)$ & $2.29(1.46-3.11)$ & \\
\hline Secondary school & 358 & $0.44(0.36-0.52)$ & $1.79(1.62-1.97)$ & $2.23(2.04-2.43)$ & \\
\hline \multicolumn{6}{|l|}{ High Education } \\
\hline College, University & 70 & $0.51(0.31-0.72)$ & $2.57(2.16-2.98)$ & $3.09(2.58-3.60)$ & \\
\hline \multicolumn{6}{|l|}{ Relationship status } \\
\hline Not dating & 262 & $0.43(0.33-0.53)$ & $1.84(1.64-2.03)$ & $2.26(2.03-2.49)$ & \multirow{3}{*}{$\begin{array}{l}P=0.037^{*} \\
F(3,445)=2.86^{\mathrm{a}}\end{array}$} \\
\hline Dating, no steady partner & 96 & $0.40(0.24-0.55)$ & $1.81(1.42-2.20)$ & $2.21(1.77-2.65)$ & \\
\hline $\begin{array}{l}\text { Dating, steady partner } \\
\text { living or not living } \\
\text { together }\end{array}$ & 82 & $0.56(0.39-0.73)$ & $2.20(1.85-2.54)$ & $2.76(2.34-3.17)$ & \\
\hline Married & 9 & $0.89(0.18-1.60)$ & $2.78(1.71-3.85)$ & $3.67(2.65-4.68)$ & \\
\hline
\end{tabular}

$* * P<0.01 ; * P<0.05$

${ }^{\text {a }}$ Significance by Welch $F$ test

${ }^{\mathrm{b}}$ Significance by ANOVA $F$ test 
Table 3 Associations between predictor variables and attitudes towards HPV vaccination

\begin{tabular}{|c|c|c|c|c|c|c|c|c|c|c|c|c|}
\hline \multirow[t]{4}{*}{ Predictor variables } & \multicolumn{12}{|c|}{ Attitude item } \\
\hline & \multicolumn{12}{|c|}{ Odds ratio ( $95 \%$ confidence interval) } \\
\hline & \multicolumn{3}{|c|}{ Want to be vaccinated } & \multicolumn{3}{|c|}{ Perceived not at risk } & \multicolumn{3}{|c|}{$\begin{array}{l}\text { Doubt the efficacy and } \\
\text { safety }\end{array}$} & \multicolumn{3}{|c|}{ Perceived shame } \\
\hline & OR & $\mathrm{CI}$ & $P$ & OR & $\mathrm{CI}$ & $P$ & OR & $\mathrm{CI}$ & $P$ & OR & CI & $P$ \\
\hline Age & 1.01 & $0.90-1.12$ & 0.93 & 0.96 & $0.84-1.09$ & 0.50 & 0.93 & $0.83-1.05$ & 0.23 & 0.83 & $0.73-0.95$ & $0.006^{* *}$ \\
\hline \multicolumn{13}{|l|}{ Ethnicity } \\
\hline $\begin{array}{l}\text { Malay } \\
\text { Non-Malay }\end{array}$ & $\begin{array}{l}1.00 \\
0.75\end{array}$ & $0.50-1.13$ & 0.18 & $\begin{array}{l}1.00 \\
1.52\end{array}$ & $0.93-2.50$ & 0.10 & $\begin{array}{l}1.00 \\
1.20\end{array}$ & $0.77-1.85$ & 0.43 & $\begin{array}{l}1.00 \\
1.31\end{array}$ & $0.80-2.13$ & 0.29 \\
\hline \multicolumn{13}{|l|}{ Educational level } \\
\hline $\begin{array}{l}\text { Low Education } \\
\text { High education }\end{array}$ & $\begin{array}{l}1.00 \\
0.45\end{array}$ & $0.15-1.39$ & 0.16 & $\begin{array}{l}1.00 \\
4.50\end{array}$ & $0.59-34.36$ & 0.15 & $\begin{array}{l}1.00 \\
0.82\end{array}$ & $0.30-2.23$ & 0.70 & $\begin{array}{l}1.00 \\
1.23\end{array}$ & $0.34-4.39$ & 0.75 \\
\hline \multicolumn{13}{|l|}{ Relationship status } \\
\hline $\begin{array}{l}\text { Not dating } \\
\text { Dating }\end{array}$ & $\begin{array}{l}1.00 \\
0.95\end{array}$ & $0.63-1.42$ & 0.80 & $\begin{array}{l}1.00 \\
0.86\end{array}$ & $0.53-1.40$ & 0.55 & $\begin{array}{l}1.00 \\
1.34\end{array}$ & $0.87-2.05$ & 0.18 & $\begin{array}{l}1.00 \\
1.39\end{array}$ & $0.87-2.24$ & 0.17 \\
\hline $\begin{array}{l}\text { Knowledge of HPV } \\
\text { and genital warts }\end{array}$ & 0.99 & $0.77-1.26$ & 0.94 & 0.91 & $0.67-1.24$ & 0.56 & 1.14 & $0.89-1.46$ & 0.31 & 0.76 & $0.54-1.06$ & 0.11 \\
\hline $\begin{array}{l}\text { Knowledge of } \\
\text { cervical screening } \\
\text { and cervical cancer } \\
\text { risk factors }\end{array}$ & 1.17 & $1.03-1.33$ & $0.013 *$ & 1.08 & $0.94-1.24$ & 0.29 & 1.04 & $0.91-1.18$ & 0.56 & 1.04 & $0.90-1.19$ & 0.62 \\
\hline $\begin{array}{l}\text { Knowing of } \\
\text { someone who has } \\
\text { had cervical cancer }\end{array}$ & 1.84 & $0.59-5.77$ & 0.29 & 1.30 & $0.44-3.84$ & 0.63 & 1.30 & $0.49-3.42$ & 0.60 & 1.21 & $0.41-3.57$ & 0.73 \\
\hline
\end{tabular}

Model 1 want to be vaccinated; Hosmer and Lemeshow test, $\chi^{2}(8)=7.942, p=0.439$; Cox and Snell $R^{2}=0.029$; Nagelkerke $R^{2}=0.040$. Model 2 perceived not at risk; Hosmer and Lemeshow test, $\chi^{2}(8)=3.991, p=0.858$; Cox and Snell $R^{2}=0.019$; Nagelkerke $R^{2}=0.030$. Model 3 doubt efficacy and safety; Hosmer and Lemeshow test, $\chi^{2}(8)=15.895, p=0.044$; Cox and Snell $R^{2}=0.012$; Nagelkerke $R^{2}=0.018$. Model 4 perceived shame; Hosmer and Lemeshow test, $\chi^{2}(8)=5.849, p=0.664$; Cox and Snell $R^{2}=0.029$; Nagelkerke $R^{2}=0.045$

$* * P<0.01 ; * P<0.05$

\section{Discussion}

The findings demonstrate knowledge deficits among the sample of rural women. The knowledge deficit had been expected and was similar to other findings in women in rural areas. The proportion which reported to have heard about HPV was low (11.6\%) compared to other studies on rural women published in the Western literature. A total of $24 \%$ of women from a rural area of the southern USA reported having heard of HPV [30]. A Canadian study reported $15 \%$ had heard about HPV in a sample of combination of urban and rural populations [31]. Nevertheless, a recently published study of rural women in China reported even a lower rate, with only 9.3\% having knowledge about HPV [32]. In Australia, the first country worldwide to commence a government funded universal HPV vaccine program, a high level of HPV awareness was reported, with $51.2 \%$ of women in Victoria reported to have heard of HPV [33].

Those that were aware of HPV and HPV vaccine had only recently heard about HPV and the new preventive vaccine. The vast majority had little knowledge about the etiological relationship of oncogenic types of HPV to cervical cancer. Likewise, benefits of the vaccine were also poorly understood. The research findings revealed widespread lack of knowledge, as manifested in the extremely low knowledge scores in all categories. These knowledge deficits need to be addressed since lack of knowledge may lead to low risk perception and low acceptability of the vaccine. Thus, the findings suggest the urgent need for public education which explicitly addresses knowledge deficits among the rural populations.

In our previous study on a sample of Malaysian female university women using the same questionnaire [34], the proportion which had heard of HPV (21.7\%) and the newly HPV vaccine $(10.3 \%)$ was higher than in the rural women in the present study. Likewise, the mean total knowledge score among the university sample was also higher (3.25 \pm 2.41). Additionally, our physicians reported that vaccine uptake among the low-educated women was poorer than among women with a higher education level, and perceived cost was the main barrier to vaccine uptake [35]. Thus, 
these results suggest that more emphasis should be placed on educating the disadvantaged socio-economic background and less-educated population (below tertiary level), as indicated in other studies [26, 32]. In the present study, poor knowledge was not limited to those not in a dating relationship. Those who were currently dating also demonstrated knowledge deficits.

The present study has shed light on interest among rural Southeast Asian women in receiving the HPV vaccine. The intention to receive the vaccine $(65 \%)$ is similar to that found in a recent study on rural Southern American women [11]. It is of interest to note that our study was conducted approximately 2 years after the release of the HPV vaccine in our market and before the widespread media coverage. Other studies conducted on urban or a combination of urban and rural populations showed that the intention for vaccination ranged from as high as $96 \%$ [36] to as low as $50 \%$ [37]. On the whole, in studies with both urban and rural participants, the rural participants were significantly more likely to indicate a negative intention to get the HPV vaccine compared to their urban counterparts $[32,38]$.

The majority learned about the vaccine from their friends more than from the public media. This indicates a probable lack of media publicity. Lack of media publicity about the new HPV vaccine was also reported in our previous focus group findings $[23,25]$. This may indicate that the media in general do not play an important role in health information dissemination for local residents, and that outreach activities would perhaps be more effective for women living in rural communities. It has been reported that community outreach intervention strategies using community or opinion leaders as capacity builders may be beneficial [30, 39].

Numerous studies concluded that recommendations from health professionals strongly correlated with intent to receive the HPV vaccine [40]. Although the current study did not investigate the association between physician recommendation and intention to vaccination, the high proportion (74\%) of participants indicated willingness to accept the vaccine if a physician would recommend vaccination. This coupled with our previous qualitative findings $[23,25]$ suggest that health care professionals' recommendations and endorsement of the vaccine are important to initiate vaccine uptake. Thus, health professionals play a pivotal role to educate and introduce the HPV vaccine to women who present themselves in the health care settings.

Approximately $20 \%$ of the rural women felt embarrassed about receiving an STI vaccine, as contrasted with $11 \%$ among the university women sample [33]. The issue of embarrassment was brought up by many women, particularly by the Muslims, less educated and rural women in our previous focus group studies [23, 25]. It is crucial to destigmatize the HPV vaccine by having health communi- cation campaigns in rural areas. In our previous study [35], the high cost of the vaccine restricted vaccination uptake among the poor and underserved women in Malaysia. Therefore, there is also a need to establish a vaccine assistance program for women in economically underserved regions.

A key limitation of the study was the non-probability convenience sampling method which may have introduced selection bias. The sample may not be representative of rural young women in Malaysia. Nevertheless, this study provides the basis for future large-scale, nationwide studies assessing knowledge, attitudes, and acceptability in rural communities. Additionally, all measures were based on participants' self-reports; thus, high intent to receive the vaccine despite poor knowledge may be the result of the respondents' tendency to give socially desirable responses. Finally, the evaluation of rural women's knowledge and perceptions may not be accurate, since these women may not have fully understood what they were asked.

\section{Conclusion}

As knowledge about cervical screening and risk factors of cervical cancer was associated with acceptance or rejection of HPV vaccination, it is important to overcome knowledge deficits among young women in rural settings in Malaysia.

Acknowledgment The study was not funded in whole or in part by any research grant or funding body. Sincere thanks and appreciation to Hajjariah bt Jaapar, Nur Farhana bt Mohd Rashidi, Izzati Nadhirah bt Mohamad, and Roszita bt Mokhtar for helping in data collection.

\section{References}

1. Parkin DM, Bray F, Ferlay J, Pisani P. Estimating the world cancer burden: Globocan 2000. Int J Cancer. 2001;94(2):153-6.

2. Lim GCC, Halimah Y (Eds.) Second Reports of the National Cancer Registry. Cancer Incidence in Malaysia 2003. Kuala Lumpur. National Cancer Registry, 2004.

3. Walboomers JMM, Jacobs MV, Manos MM, et al. Human papillomavirus is a necessary cause of invasive cervical cancer worldwide. J Pathol. 1999;189:12-9.

4. Munoz N, Bosch FX, de Sanjos S, et al. Epidemiologic classification of human papillomavirus types associated with cervical cancer. N Engl J Med. 2003;384:518-27.

5. Castellsagué X, Díaz M, de Sanjosé S, et al. Worldwide human papillomavirus etiology of cervical adenocarcinoma and its cofactors: implications for screening and prevention. J Natl Cancer Inst. 2006;98:303-15.

6. Kane MA, Sherris J, Coursaget P, Aguado T, Cutts F. HPV vaccine use in the developing world. Vaccine. 2006;24(3):S132-9.

7. Agosti JM, Goldie SJ. Introducing HPV vaccine in developing countries - key challenges and issues. N Engl J Med. 2007;356 (19):1908-10.

8. Waller J, McCaffery K, Forrest S, Szarewski A, Cadman L, Wardle J. Awareness of human papillomavirus among women 
attending a well women clinic. Sex Trans Infect. 2003;79 (4):320-2.

9. Lambert EC. College student's knowledge of human papillomavirus and effectiveness of a brief educational intervention. J Am Board Fam Pract. 2001;14(3):178-83.

10. Ramirez JE, Ramos DM, Clayton L, Kanowitz S, Moscicki AB. Genital human papillomavirus infection: knowledge, perception of risk, and actual risk in a nonclinic population of young women. J Womens Health. 1997;6(1):113-21.

11. Hopenhayn C, Christian A, Christian WJ, Schoenberg NE. Human papillomavirus vaccine: knowledge and attitudes in two Appalachian Kentucky counties. Cancer Causes Control. 2007;18:62734.

12. Mays RM, Sturm LA, Zimet GD. Parental perspectives on vaccinating children against sexually transmitted infections. Soc Sci Med. 2004;58:1405-13.

13. Dempsey AF, Zimet GD, Davis RL, Koutsky L. Factors that are associated with parental acceptance of human papillomavirus vaccines: a randomized intervention study of written information about HPV. Pediatrics. 2006;117(5):1486-93.

14. Smailyte G, Kurtinaitis J. Cancer mortality differences among urban and rural residents in Lithuania. BMC Public Health. 2008;8:56.

15. Palacio-Mejía LS, Rangel-Gómez G, Hernández-Avila M. Lazcano-Ponce E cervical cancer, a disease of poverty: mortality differences between urban and rural areas in Mexico. Salud Pública Méx. 2003;45(3):S315-25.

16. Hopenhayn C, Bush H, Christian A, Shelton BJ. Comparative analysis of invasive cervical cancer incidence rates in three Appalachian states. Prev Med. 2005;41(5-6):859-64.

17. Elder JP, Castro FG, de Moor C, et al. Differences in cancerrisk-related behavior in Latino and Anglo adults. Prev Med. 1991;20:751-63.

18. The National Cancer Institute Cancer Screening Consortium for Underserved Women. Breast and cervical cancer screening among underserved women. Baseline survey results from six studies. Arch Fam Med. 1995;4:617-24.

19. Akers AY, Newmann SJ, Smith JS. Factor underlying disparities in cervical cancer incidence, screening and treatment in the United States. Curr Probl Cancer. 2007;31(3):157-81.

20. Gerend MA, Lee SC, Shepherd JE. Predictors of human papillomavirus vaccination acceptability among underserved women. Sex Transm Dis. 2007;34:468-71.

21. Fazekas KI, Brewer NT, Smith JS. HPV vaccine acceptability in a rural, Southern area. J Womens Health. 2008;17(4):539-48.

22. Sperber NR, Brewer NT, Smith JS. Influence of parent characteristics and disease outcome framing on HPV vaccine acceptability among rural, Southern women. Cancer Causes Control. 2008;19 (1):115-18.

23. Wong LP. Preventing cervical cancer through human papillomavirus vaccination: perspective from focus groups. J Low Genit Tract Dis. 2009;13(2):85-93.

24. Sam IC, Wong LP, Rampal S, et al. Maternal acceptance of human papilomavirus vaccine in Malaysia. J Adolesc Health. 2009;44 (6):610-12.
25. Wong LP. Young multiethnic women's attitudes toward the HPV vaccine and HPV vaccination. Int J Gynecol Obstet. 2008;103 (2):131-5.

26. Halcomb B, Bailey JM, Crawford K, Ruffin 4th MT. Adults' knowledge and behaviors related to human papillomavirus infection. J Am Board Fam Pract. 2004;17(1):26-31.

27. Giles M, Garland S. A study of women's knowledge regarding human papillomavirus infection, cervical cancer and human papillomavirus vaccines. Aust NZ J Obstet Gynaecol. 2006;46:311-15.

28. Wang JF, Simoni PS, Wu Y. Human papillomavirus (HPV) in rural adolescent females: knowledge, protected sex, and sexual risk behaviors. Online Journal of Rural Nursing and Health Care 2006; 6(1) [online]. Available: http://www.rno.org/journal/index. $\mathrm{php} /$ online-journal/article/view/34

29. Philips Z, Johnson S, Avis M, Whynes DK. Human papillomavirus and the value of screening: young women's knowledge of cervical cancer. Health Educ Res. 2003;18(3):318-28.

30. Cates JR, Brewer NT, Fazekas KI, Mitchell CE, Smith JS. Racial differences in HPV knowledge, HPV vaccine acceptability, and related beliefs among rural, southern women. J Rural Health. 2009;25(1):93-7.

31. Sauvageau C, Duval B, Gilca V, Lavoie F, Ouakki M. Human papilloma virus vaccine and cervical cancer screening acceptability among adults in Quebec, Canada. BMC Public Health. 2007;7(1):304.

32. Li J, Li LK, Ma JF, Wei LH, Niyazi M, Li CQ, et al. Knowledge and attitudes about human papillomavirus (HPV) and HPV vaccines among women living in metropolitan and rural regions of China. Vaccine. 2009;27(8):1210-15.

33. Pitts MK, Dyson SJ, Rosenthal DA, Garland SM. Knowledge and awareness of human papillomavirus (HPV): attitudes towards HPV vaccination among a representative sample of women in Victoria, Australia. Sex Health. 2007;4(3):177-80.

34. Wong LP, Sam IC. Ethnically diverse female university students' knowledge and attitudes toward human papillomavirus (HPV), HPV vaccination and cervical cancer. Eur J Obstet Gyn Reprod Biol. 2010;148:90-5.

35. Wong LP. Physicians' experiences with HPV vaccine delivery: evidence from developing country with multiethnic populations. Vaccine. 2009;27:1622-7.

36. Baykal C, Al A, Uğur MG, Cetinkaya N, Attar R, Arioglu P. Knowledge and interest of Turkish women about cervical cancer and HPV vaccine. Eur J Gynecol Oncol. 2008;29:76-9.

37. Donders GG, Gabrovska M, Bellen G, et al. Knowledge of cervix cancer, human papilloma virus (HPV) and HPV vaccination at the moment of introduction of the vaccine in women in Belgium. Arch Gynecol Obstet. 2008;277:291-8.

38. Lenselink CH, Schmeink CE, Melchers WJG, et al. Young adults and acceptance of the human papillomavirus vaccine. Public Health. 2008;122:1295-301.

39. Lisovicz N, Johnson RE, Higginbotham J, et al. The Deep South Network for cancer control-building a community infrastructure to reduce cancer health disparities. Cancer. 2006;107(S8):1971-9.

40. Brewer NT, Fazekas KI. Predictors of HPV vaccine acceptability: a theory-informed, systematic review. Prev Med. 2007;45:107-14. 